\title{
A Multicentre, Efficacy and Safety Study of Methotrexate to Increase Response Rates in Patients With Uncontrolled Gout Receiving
} Pegloticase (MIRROR): 12-Month Efficacy, Safety, Immunogenicity, and Pharmacokinetic Findings of an Open-label Study

John Botson ( $\sim$ jbotson@opaak.com )

Orthopedic Physicians Alaska https://orcid.org/0000-0002-9552-3351

John RP Tesser

Arizona Arthritis \& Rheumatology Associates

Ralph Bennett

Arizona Arthritis \& Rheumatology Associates

Howard M Kenney

Arthritis Northwest, PLLC

Paul M Peloso

Horizon Therapeutics plc

Katie Obermeyer

Horizon Therapeutics plc

Yang Song

Horizon Therapeutics plc

Brian LaMoreaux

Horizon Therapeutics plc

Lin Zhao

Horizon Therapeutics plc

Yan Xin

Horizon Therapeutics plc

Jason Chamberlain

Horizon Therapeutics plc

Srini Ramanathan

Horizon Therapeutics plc

Michael E Weinblatt

Division of Rheumatology, Inflammation and Immunity, Brigham and Women's Hospital Jeff Peterson 
Western Washington Medical Group Arthritis Clinic

\section{Research article}

Keywords: pegloticase, methotrexate, gout, tophi

Posted Date: October 26th, 2021

DOl: https://doi.org/10.21203/rs.3.rs-951365/v1

License: (9) This work is licensed under a Creative Commons Attribution 4.0 International License. Read Full License 


\section{Abstract}

Background: Publications suggest immunomodulation co-therapy improves responder rates in uncontrolled/refractory gout patients undergoing pegloticase treatment. The MIRROR open-label trial showed a 6-month pegloticase+methotrexate co-therapy responder rate of $79 \%$, compared to an established $42 \%$ pegloticase monotherapy responder rate. Longer-term efficacy/safety data are presented here.

Methods: Uncontrolled gout patients (serum urate $[S U] \geq 6 \mathrm{mg} / \mathrm{dL}$ and $\mathrm{SU} \geq 6 \mathrm{mg} / \mathrm{dL}$ despite uratelowering therapy [ULT], ULT intolerance, or functionally-limiting tophi) were included. Patients with immunocompromised status, G6PD deficiency, severe kidney disease, or methotrexate contraindication were excluded. Oral methotrexate (15 mg/week) and folic acid ( $1 \mathrm{mg} /$ day) were administered 4-weeks before and during pegloticase therapy. Twelve-month responder rate ( $\mathrm{SU}<6 \mathrm{mg} / \mathrm{dL}$ for $\geq 80 \%$ during Month 12), 52-week change from baseline in SU, and extended safety were examined. Efficacy analyses were performed for patients receiving $\geq 1$ pegloticase infusion. PK/anti-drug antibodies (ADAs) were examined and related to efficacy/safety findings.

Results: Fourteen patients were included (all male, $49.3 \pm 8.7$ years, $13.8 \pm 7.4$ year gout history, pretherapy SU: $9.2 \pm 2.5 \mathrm{mg} / \mathrm{dL}$ ). Three patients were non-responders and discontinued study treatment before 24-weeks, one patient exited the study per-protocol at 24-weeks (enrolled prior to treatment extension amendment), and 10 remained in study through Week 52. Of the 10, 8 completed 52-weeks of pegloticase+methotrexate and were 12-month responders. The remaining two discontinued pegloticase+methotrexate at Week 24 (met treatment goals) and stayed in study under observation (allopurinol prescribed at physicians' discretion); one remained a responder at 12-months. At 52-weeks, change from baseline in SU was $-8.2 \pm 4.1 \mathrm{mg} / \mathrm{dL}$ (SU: $1.1 \pm 2.4 \mathrm{mg} / \mathrm{dL}, \mathrm{n}=10$ ). Gout flares were common early in treatment but progressively decreased while on therapy (Weeks 1-12: 13/14 [92.9\%], Weeks 36-52: $2 / 8$ [25.0\%]). One patient recovered from sepsis (serious AE). Two non-responders developed high ADA titres; fewer patients had trough concentrations $\left(\mathrm{C}_{\mathrm{min}}\right)$ below quantitation limit $(\mathrm{BQL})$ and median $\mathrm{C}_{\text {min }}$ was higher $(1.03 \mathrm{mg} / \mathrm{mL}$ vs. BQL) than in pegloticase monotherapy trials.

Conclusions: Methotrexate+pegloticase co-therapy was well-tolerated over 12-months, with sustained SU lowering, progressive gout flare reduction, and no new safety concerns. Antibody/PK findings suggest methotrexate attenuates ADA formation, coincident with higher treatment response rates.

Trial registration: ClinicalTrials.gov: NCT03635957, registered 17 August 2018, https://clinicaltrials.gov/ct2/show/NCT03635957

\section{Background}

Gout is a common, inflammatory arthritis caused by monosodium urate deposition in the setting of chronically elevated serum urate levels ( $\mathrm{SU}>6 \mathrm{mg} / \mathrm{dL}$ ). Monosodium urate crystals cause chronic systemic inflammation, even between acute gout flares [1, 2]. Additionally, hyperuricemia has been linked 
to multiple comorbidities, including hypertension $[3,4]$, cardiovascular disease [5-9], diabetes $[3,10]$, kidney disease $[11,12]$, and a higher mortality rate $[9,13,14]$. Gout treatment guidelines recommend maintaining SU below at least $6 \mathrm{mg} / \mathrm{dL}[15,16]$, but some patients are unable to meet this target because of urate-lowering therapy (ULT) under-utilization [17], patient non-compliance [18, 19], ULT intolerance, or ULT inefficacy [20]. As a result, an estimated $10 \%$ of patients may develop uncontrolled gout [21], which markedly impairs patient quality of life and physical functioning [22].

Pegloticase, a pegylated uricase enzyme, is an FDA approved therapy for adult patients with uncontrolled gout and is highly effective in lowering SU [23]. The recombinant enzyme rapidly lowers SU by converting uric acid to allantoin, a water-soluble molecule that is readily excreted by the kidneys. Though initial response to treatment is robust, many patients develop anti-drug antibodies (ADAs) to the therapy and are unable to complete a full course of treatment. Clinical trials of pegloticase monotherapy found a $42 \%$ treatment responder rate (responder defined as patient with $\mathrm{SU}<6.0 \mathrm{mg} / \mathrm{dL}$ for $80 \%$ of the time or longer during both Months 3 and 6 of therapy), with approximately one-quarter $(26 \%)$ of patients experiencing infusion reactions (IRs) when a pre-infusion monitoring protocol for SU was not followed [23, 24]. Antipegloticase antibodies that develop following pegloticase exposure are associated with both loss of urate lowering effect, through increased pegloticase clearance, and a higher risk of IRs [25-27]. Starting in 2017, successes with immunomodulation co-therapy have been reported in the real-world setting with methotrexate $[28,29]$ and leflunomide [30] and in a clinical trial setting with azathioprine (TRIPLE openlabel) [31], methotrexate (MIRROR open-label trial) [32], and mycophenolate mofetil in a randomized placebo controlled clinical trial (RECIPE trial) [33]. Likely related to these reports, immunomodulation use with pegloticase is increasing in the United States [34]. In 2015, approximately $2 \%$ of patients treated with pegloticase were also treated with immunomodulation, whereas in $2019,15 \%$ of new pegloticase patients were also treated with immunomodulating co-therapy [34]. Initial MIRROR open-label clinical trial reports describe 6-month outcomes [32], but longer-term safety and efficacy have not yet been published. Here, 12-month efficacy outcomes are described, along with pharmacokinetic (PK) and immunogenicity findings.

\section{Methods}

\section{Study population}

This study population has been fully described elsewhere [32]. Briefly, patients with uncontrolled gout aged 18-65 years were considered for trial enrolment. Patients were said to have uncontrolled gout if their SU level was $\geq 6 \mathrm{mg} / \mathrm{dL}$ at Screening and at least one of the following was true: they were unable to maintain $\mathrm{SU}<6 \mathrm{mg} / \mathrm{dL}$ on an oral ULT, they had intolerance to their current ULT, or tophaceous deposits that limited patient functionality were present (detected clinically or with dual-energy computed tomography [DECT]). Key exclusion criteria included serious acute bacterial infection $<2$ weeks prior to Screening, severe chronic/recurrent bacterial infection, immunocompromised status, glucose-6phosphate dehydrogenase (G6PD) deficiency (tested at Screening), severe chronic renal impairment 
(glomerular filtration rate $[\mathrm{GFR}]<25 \mathrm{~mL} / \mathrm{min} / 1.73 \mathrm{~m}^{2}$ or currently on dialysis), or liver disease (alanine aminotransferase $[\mathrm{ALT}]$ or aspartate aminotransferase $[\mathrm{AST}]>3$ times upper limit).

\section{Study medications}

All enrolled patients were scheduled to receive 4 weeks of oral methotrexate ( $15 \mathrm{mg} /$ week, run-in period) followed by treatment with both pegloticase ( $8 \mathrm{mg}$ infusion every 2 weeks) and methotrexate $(15 \mathrm{mg}$ orally every week) for up to 52 weeks (treatment period). The original protocol included a 24-week treatment period, but a protocol amendment extended the treatment period to 52 weeks. Patients also received $1 \mathrm{mg} /$ day oral folic acid during both the run-in and treatment periods. All patients were required to begin gout flare prophylaxis (colchicine, non-steroidal anti-inflammatory drugs [NSAIDs], and/or lowdose prednisone [ $\leq 10 \mathrm{mg} /$ day] as chosen by the treating physician) at least 1 week prior to initiating pegloticase, continuing flare prophylaxis per American College of Rheumatology guidelines [15]. When they did occur, flares were managed with NSAIDs, colchicine, corticosteroids, and intraarticular steroid injections at the treating physician's discretion.

Patients were administered standard IR prophylaxis prior to each pegloticase infusion. This included oral fexofenadine (60 or $180 \mathrm{mg}$ ) the day before and morning of infusion, acetaminophen (1000 $\mathrm{mg}$ ) the morning of infusion, and intravenous glucocorticoid (200 mg hydrocortisone or $125 \mathrm{mg}$ methylprednisolone) immediately prior to each infusion. An SU monitoring protocol [24] was implemented to decrease the risk of IRs. Patients discontinued pegloticase/methotrexate co-therapy if they had two consecutive SU measurements above $6 \mathrm{mg} / \mathrm{dL}$ after Week 2.

\section{Study procedures}

Study procedures from Screening through Month 6 have been previously described [32]. Briefly, Screening included study eligibility confirmation and patient medical and surgical history (including concomitant medications), physical examination, gout assessment (including flare history), and laboratory testing (SU measurement, haematology, clinical chemistry). After eligibility was confirmed, patients began the 4-week methotrexate run-in period (-4 weeks) within 2 weeks of Screening and returned for physical, laboratory, gout flare, and safety assessment at Week -2 .

Pegloticase therapy was initiated on Day 1 (methotrexate and folic acid continued during pegloticase treatment) and had a maximum duration of 52 weeks. Patients returned every 2 weeks for follow-up assessment (concomitant medication update, physical examination) and pegloticase infusion (see Additional file 1 for further detail). Safety assessments at each study visit included AE evaluation and blood/urine sampling for laboratory measurement and SU monitoring. The published Rheumatology Common Terminology Criteria for Adverse Events (CTCAE) grading system ( $1=$ mild, $2=$ moderate, $3=$ severe, 4 = life-threatening) was used to determine AE severity [35]. When gout flares were documented, severity was assessed using a standardized flare grading system based on joint pain, joint swelling, and pain levels at rest [36].

\section{Pharmacokinetics and anti-drug antibodies}


Blood samples for pegloticase PK evaluations were collected before and after pegloticase infusion on Day 1 and at Weeks 4, 8, 22, and 36. Samples were also collected before pegloticase infusion at Weeks $10,14,18,22$, and 52. Pre-infusion blood samples were collected from all patients to evaluate the immunogenicity of pegloticase by measuring both anti-PEG and anti-uricase immunoglobulin $\mathrm{G}$ (IgG) antibodies (ADAs) at Day 1 and Weeks $2,4,6,8,10,14,18,22,24,36$, and 52 . A small subset of patients had additional visits at Weeks 1 and 7 (non-infusion visits), during which blood samples were collected for both PK and antibody evaluations. Pre-infusion blood samples were also collected to measure methotrexate polyglutamate(s) (MTX-PGs 1 to 5 ) in red blood cells at Day 1 and Weeks 4, 8, 22, 24 and 36.

\section{Bioanalytical assays}

Pegloticase concentration in serum was measured using a validated enzymatic/fluorescence assay (Charles River Laboratories, Senneville, Quebec, Canada). The calibration range was 0.6 to $10 \mu \mathrm{g} / \mathrm{mL}$. ADAs were measured using a validated enzyme linked immunosorbent assay (anti-pegloticase/PEG, antiuricase; Precision for Medicine, Redwood City, CA, USA). The drug tolerance level was $<0.02 \mu \mathrm{g} / \mathrm{mL}$ in neat serum. Measurements of MTX-PG1 to 5 red blood cell concentrations were performed by Exagen Inc. (AVISE ${ }^{\circledR}$ MTX test; Vista, CA, USA) using a liquid chromatography-mass spectrometry method [37].

\section{Pegloticase pharmacokinetic analysis}

Serum pegloticase concentration data were analyzed using two methods to evaluate the impact of methotrexate co-therapy on pegloticase PK. First, pegloticase exposures (median observed peak concentration $\left[\mathrm{C}_{\max }\right]$ and trough concentration $\left[\mathrm{C}_{\mathrm{min}}\right]$ across visits) with methotrexate from the current study were compared to the observed values in historical monotherapy Phase 3 studies (C0405 and C0406) $[23,26]$. Second, the observed pegloticase concentrations with methotrexate co-therapy in the current study were overlaid against the $90 \%$ prediction band of pegloticase concentrations following monotherapy based on the population PK model from the Phase 3 data [38]. Pharmacokinetic profiles of pegloticase following $8 \mathrm{mg}$ IV infusion every 2 weeks for a total of 12 infusions were simulated for 400 patients with an average body surface area of $2.12 \mathrm{~m}^{2}$ (percent coefficient of variation $=13.3 \%$ ). The population PK simulations were done using NONMEM 7.4 (ICON Development Solutions, Ellicott City, MD, USA).

\section{Statistical Methods}

The primary efficacy endpoint was the proportion of responders during Month 6 (SU $<6 \mathrm{mg} / \mathrm{dL}$ for $\geq 80 \%$ of Month 6) and has been fully reported elsewhere [32]. Secondary and exploratory efficacy objectives described here include proportion of treatment responders ( $\mathrm{SU}<6 \mathrm{mg} / \mathrm{dL}$ during $\geq 80 \%$ of examined time) during Months 3, 9, and 12, overall response rate during Months 3 and 6 combined, and mean change from baseline in SU at Weeks 14, 24, 36, and 52. A sample size of 12-16 patients was planned, which would demonstrate a statistically greater response rate over pegloticase monotherapy if at least 10/13 $(77 \%)$ patients were responders for the primary endpoint (proportion of pegloticase responders during 
Month 6 in pegloticase pivotal trials [43.5\%] [23]), based on an exact test for proportions with a 5\% type 1 error. Longer-term (9 month, 12 month) efficacy endpoints do not have established historical comparators.

All efficacy and safety analyses were performed on the modified intent-to-treat (mITT) population, defined as all patients who received at least 1 dose of pegloticase. Continuous variables are summarized by visit as mean \pm standard deviation and categorical variables are summarized as $\mathrm{n}(\%)$. SU values below quantification limits (BQL) were set to zero for analyses. Safety analyses were also performed on data collected during the run-in period using the ITT population, defined as all patients who received at least 1 dose of methotrexate. The incidence and titre of positive ADAs (anti-PEG and anti-uricase) are summarized by visit.

\section{Results}

Seventeen patients were screened for study eligibility between September 2018 and April 2019. Fifteen patients began the methotrexate run-in period and made up the ITT population. Fourteen completed the entire 4-week run-in period (1 patient was lost to follow-up after Week -2), began methotrexate/pegloticase co-therapy, and made up the mITT population through Week 24. As already reported, 11 of 14 completed 24-weeks of methotrexate+pegloticase co-therapy for a 6-month responder rate of 79\% (95\% Cl: 49.2-95.3\%; 3 patients discontinued pegloticase prior to 24-weeks because of SU rise) [32]. Of the 11 patients remaining in study at Week 24, 8 continued therapy through Week 52, 2 met gout treatment goals at Week 24 discontinuing study treatment but remaining in the study for observation through Week 52, and 1 completed the study at Week 24 (enrolled prior to protocol amendment that extended treatment). One of the patients who met treatment goals at Week 24 initiated allopurinol at the treating investigator's discretion at Week 24 (300 mg/day for 1 week, 600 mg/day for 2 weeks, then $300 \mathrm{mg} /$ day thru Week 52) and the other patient started allopurinol at Week 26 (150 mg/day for 21 weeks then 300 mg/day thru Week 52; see Additional file 2). The patient who completed the study at Week 24 was not included in analyses beyond 24 weeks (i.e., mITT N = 13 after Week 24).

Table 1 summarizes mITT patient characteristics at baseline. Briefly, patients were $49.3 \pm 8.7$ years of age and had an average gout history of $13.8 \pm 7.4$ years. Prior to initiating pegloticase, SU averaged $9.2 \pm$ $2.5 \mathrm{mg} / \mathrm{dL}$ and 13 patients (93\%) had clinically evident tophi. 
Table 1

Baseline characteristics for the modified intent-to-treat population ( $N=14)$

\begin{tabular}{|lc|}
\hline Age, mean \pm SD, years & $49.3 \pm 8.7$ \\
\hline Male sex, $\mathrm{n}(\%)$ & $14(100)$ \\
\hline Race, $\mathrm{n}(\%)$ & $12(85.7)$ \\
\hline Asian & $2(14.3)$ \\
\hline Body mass index (BMI), mean $\pm \mathrm{SD}, \mathrm{kg} / \mathrm{m}^{2}$ & $33.9 \pm 7.0$ \\
\hline Smoking status, $\mathrm{n}(\%)$ & \\
\hline Never & $4(28.6)$ \\
\hline Current & $5(35.7)$ \\
\hline Former & $5(35.7)$ \\
\hline Gout characteristics & \\
\hline Time since first gout diagnosis, mean \pm SD, years & $13.8 \pm 7.4$ \\
\hline Number of gout flares in the 12 months prior to Screening, mean \pm SD & $10.8 \pm 8.5$ \\
\hline History of tophi, $\mathrm{n}(\%)$ & $13(92.9)$ \\
\hline Baseline serum urate, mean \pm SD, mg/dL & $9.2 \pm 2.5$ \\
\hline
\end{tabular}

\section{Efficacy Outcomes}

All 8 patients who continued pegloticase/methotrexate co-therapy through Week 52 were treatment responders during Months 9 and 12. Pegloticase response rate in the mITT population was 10 of 13 patients (76.9\%) during Month 9 and 9 of 13 patients (69.2\%) during Month 12 (Table 2). Of the 4 patients who did not meet response criteria during Month 12, all had discontinued study treatment prior to (3 patients met pegloticase discontinuation criteria [1 patient each at Week 4, Week 6, and Week 10]) or at Week 24 (1 patient met treatment goals and discontinued pegloticase). After initiating pegloticase, SU rapidly decreased and was $0.0 \pm 0.0 \mathrm{mg} / \mathrm{dL}$ at Week 24 (change from baseline: $-9.3 \pm 2.8 \mathrm{mg} / \mathrm{dL}, \mathrm{n}=11$ ) and $1.1 \pm 2.5 \mathrm{mg} / \mathrm{dL}$ at Week 52 (change from baseline: $-8.2 \pm 4.1 \mathrm{mg} / \mathrm{dL}, \mathrm{n}=10$ ). In the patients remaining on pegloticase/methotrexate treatment through Week $52(n=8)$, SU was $0.0 \pm 0.0$ at Weeks 36 and 52 (change from baseline: $-9.4 \pm 3.3 \mathrm{mg} / \mathrm{dL}$; Figure 1, Table 2). In the 2 patients who remained in study, but discontinued pegloticase/methotrexate at Week 24 , SU remained $<6 \mathrm{mg} / \mathrm{dL}$ until Week 36 in the patient who initiated allopurinol at Week 24 and until Week 50 in the patient who initiated allopurinol at Week 26 (see Additional file 2). 
Table 2

Efficacy endpoints through Month 12/Week 52.

Efficacy endpoint

Maintained $\mathrm{SU}<6 \mathrm{mg} / \mathrm{dL}$ for at least $80 \%$ of the time during:

Month 3, n (\%) [95\% Cl], N=14

Month 6, n (\%) [95\% Cl], N=14

Months 3 and 6 (overall), n (\%) [95\% Cl], N=14

Month 9, n (\%) [95\% Cl], N=13

Month 12, n (\%) [95\% Cl], N=13

SU change from baseline, $\mathrm{mg} / \mathrm{dL}$, mean $\pm \mathrm{SD}$ (median [min, max])*

Week 24, mean \pm SD (median [min, max]), $n=11$

Week 36, mean \pm SD (median [min, max]), $\mathrm{n}=8^{\dagger}$

Week 52, mean \pm SD (median [min, max] $) \mathrm{n}=8^{+}$

Confidence intervals $(\mathrm{Cl})$ based on exact (Clopper-Pearson) $\mathrm{Cl}$. *includes patients remaining on treatment, values below the lower limit of detection were set to 0 , baseline is the last observation prior to the first pegloticase infusion. ${ }^{\dagger}$ Change from baseline was $-8.1 \pm 4.0 \mathrm{mg} / \mathrm{dL}$ at Week 36 and $-8.2 \pm$ $4.1 \mathrm{mg} / \mathrm{dL}$ at Week 52 for all 10 patients remaining in study through Week 52. mITT, modified intent to treat (all patients exposed to pegloticase); SU, serum urate.

\section{Pharmacokinetics}

The measured concentrations of MTX-PGs were maintained during the treatment course, suggesting compliance of MTX administration (see Additional file 3). Concomitant treatment with methotrexate generally improved pegloticase exposures, resulting in a lower proportion of patients with $\mathrm{C}_{\mathrm{min}} \mathrm{BQL}$ on methotrexate (5/14 [36\%] vs. without methotrexate 63/82 [77\%]) and higher overall $\mathrm{C}_{\text {min }}$ (median [Q1, Q3]: $1.03(\mathrm{BQL}, 1.23) \mu \mathrm{g} / \mathrm{mL}$ with methotrexate vs. BQL [BQL, BQL] without methotrexate), as well as slightly higher peak concentration $C_{\max }$ (median [Q1, Q3]: $2.11[1.65,2.59] \mu \mathrm{g} / \mathrm{mL}$ with methotrexate vs. 1.51 [BQL, 2.48] $\mu \mathrm{g} / \mathrm{mL}$ without methotrexate; Figure 2, Table 3). Consistently, pegloticase co-treatment with methotrexate resulted in more observed pegloticase concentrations above the predicted median value of pegloticase monotherapy ( $66 \%$ for the entire 14-day dosing interval and $82 \%$ for trough concentrations, Figure 3). All non-responders had pegloticase $C_{\min } B Q L$ across time points, whereas responders typically had measurable $C_{\min }$ values across most time points with the exception of two patients that only have $\mathrm{C}_{\min }$ above BQL for a few visits (Figure 2). 
Table 3

Summary of pegloticase exposure with methotrexate co-treatment (current study) and as monotherapy (calculated using pharmacokinetic findings of prior phase 3 trials $[23,26]$ ).

\begin{tabular}{|c|c|c|c|c|}
\hline & \multicolumn{2}{|l|}{$C_{\max }(\mu \mathrm{g} / \mathrm{mL})$} & \multicolumn{2}{|l|}{$C_{\min }(\mu \mathrm{g} / \mathrm{mL})$} \\
\hline & MTX+pegloticase & $\begin{array}{l}\text { Pegloticase } \\
\text { (Monotherapy trial) }\end{array}$ & MTX+pegloticase & $\begin{array}{l}\text { Pegloticase } \\
\text { (Monotherapy } \\
\text { trial) }\end{array}$ \\
\hline $\begin{array}{l}\text { Median (Q1, } \\
\text { Q3) }\end{array}$ & $2.11(1.65,2.59)$ & 1.51 (BQL, 2.48) & 1.03 (BQL, 1.23) & $\mathrm{BQL}(\mathrm{BQL}, \mathrm{BQL})$ \\
\hline $\begin{array}{l}\mathrm{BQL}, \mathrm{n} / \mathrm{N} \\
(\%)\end{array}$ & $0 / 14(0 \%)$ & 25/85 (29\%) & $5 / 14(36 \%)$ & 63/82 (77\%) \\
\hline \multicolumn{5}{|c|}{$\begin{array}{l}\mathrm{C}_{\text {max }} \text { maximum pegloticase concentration during the treatment period; } \mathrm{C}_{\min }, \text { minimum pegloticase } \\
\text { concentration during the treatment period; } \mathrm{BQL} \text {, below quantitation limit }(0.6 \mu \mathrm{g} / \mathrm{mL}) ; \mathrm{MTX} \\
\text { methotrexate }\end{array}$} \\
\hline
\end{tabular}

\section{Immunogenicity}

ADA data are consistent with the improved pegloticase efficacy and PK when coadministered with methotrexate. An increase in ADA titre was observed in 2 of 3 non-responders ( 1 patient from $<10$ at baseline to 320 at Week 4, 1 patient 40 at baseline to 640 at Week 2) and temporally corresponded with loss of pegloticase exposure and SU increase. The third non-responder received 5 pegloticase infusions prior to meeting SU discontinuation criteria. This patient was positive for anti-PEG antibody titre prior to first pegloticase dose, but negative during the Treatment period. Two of eleven responders at month 6 showed a small increase $(\leq 40)$ in anti-PEG antibody titre at a single time during pegloticase/methotrexate treatment. All other anti-PEG antibody titre measurements were either negative or no greater than baseline levels. The remaining 9 responders were considered negative for the induction of anti-PEG antibodies.

\section{Safety}

Ten patients (66.7\%) experienced an AE during the run-in period, including gout flare (5 patients), nausea (2 patients), and abdominal discomfort (2 patients, Table 3). All patients experienced one or more AEs during methotrexate/pegloticase treatment, most commonly gout flare (13 patients [92.9\%]). Patients also experienced diarrhoea, nasopharyngitis, upper respiratory tract infection, muscle strain, and arthralgia (3 patients each [21.4\%], Table 3). One SAE of bacterial sepsis secondary to cholecystitis was observed and deemed unrelated to study treatments by the reporting investigator. 
Table 4

Summary of adverse events and serious adverse events.

\begin{tabular}{|c|c|c|}
\hline & $\begin{array}{l}\text { MTX Run-In } \\
\text { ( } 4 \text { weeks) } \\
\text { ITT population ( } N= \\
\text { 15) }\end{array}$ & $\begin{array}{l}\text { Pegloticase+MTX } \\
\text { Treatment } \\
\text { (up to } 52 \text { weeks) } \\
\text { mITT population }(\mathrm{N}=14 \text { ) }\end{array}$ \\
\hline Any $A E, n(\%)$ & $10(66.7 \%)$ & $14(100 \%)$ \\
\hline Any SAE, n (\%) & $0(0.0 \%)$ & $1(7.1 \%)^{\star}$ \\
\hline \multicolumn{3}{|c|}{$\begin{array}{l}\text { AEs occurring in }>1 \text { patient in either period, } n \\
(\%)\end{array}$} \\
\hline Gout flare & $5(33.3 \%)$ & $13(92.9 \%)$ \\
\hline Diarrhoea & $1(6.7 \%)$ & $3(21.4 \%)$ \\
\hline Nasopharyngitis & $1(6.7 \%)$ & $3(21.4 \%)$ \\
\hline Upper respiratory tract infection & $0(0.0 \%)$ & $3(21.4 \%)$ \\
\hline Muscle strain & $0(0.0 \%)$ & $3(21.4 \%)$ \\
\hline Arthralgia & $0(0.0 \%)$ & $3(21.4 \%)$ \\
\hline Sinusitis & $0(0.0 \%)$ & $2(14.3 \%)$ \\
\hline Hypertension & $0(0.0 \%)$ & $2(14.3 \%)$ \\
\hline Liver function test values increased & $0(0.0 \%)$ & $2(14.3 \%)$ \\
\hline Nausea & $2(13.3 \%)$ & $1(7.1 \%)$ \\
\hline Abdominal discomfort & $2(13.3 \%)$ & $0(0.0 \%)$ \\
\hline \multicolumn{3}{|l|}{ AEs of special interest, $n(\%)$} \\
\hline Infusion reactions & - & $1(7.1 \%)^{\dagger}$ \\
\hline Anaphylaxis & - & $0(0.0 \%)$ \\
\hline Cardiovascular events & $0(0.0 \%)$ & $0(0.0 \%)$ \\
\hline Gout flare (patients with $\geq 1$ flare) & $5(33.3 \%)$ & $13(92.9 \%)$ \\
\hline Number of flares, mean \pm SD [range] & $1.2 \pm 0.5[1-2]$ & $6.5 \pm 5.8[1-20]$ \\
\hline
\end{tabular}

*Bacterial sepsis unrelated to study treatment, patient remained in study on treatment. ${ }^{\dagger}$ Mild cough after infusion 5, patient remained on therapy, completed the study at Week 24, and was a treatment responder during Month 6 . MTX, methotrexate; ITT, intent-to-treat (any patient exposed to MTX during the Run-in period); mITT, modified intent-to-treat (any patient exposed to pegloticase during the Pegloticase + MTX Treatment period). 
MTX Run-In

(4 weeks)

ITT population $(\mathrm{N}=$

15)
Pegloticase+MTX

Treatment

(up to 52 weeks)

mITT population $(\mathrm{N}=14)$

Number of flares, median 1

5

*Bacterial sepsis unrelated to study treatment, patient remained in study on treatment. ${ }^{\dagger}$ Mild cough after infusion 5, patient remained on therapy, completed the study at Week 24, and was a treatment responder during Month 6. MTX, methotrexate; ITT, intent-to-treat (any patient exposed to MTX during the Run-in period); mITT, modified intent-to-treat (any patient exposed to pegloticase during the Pegloticase + MTX Treatment period).

Infusion reaction (IR), anaphylaxis, cardiovascular events, and gout flare were AEs of special interest. A single IR was reported in one patient following the 5th pegloticase infusion (Week 8 visit). As previously detailed [32], the event was described by the investigator as a mild cough that began towards the end of the infusion and resolved without treatment after approximately 1 hour. Typical IR signs (hives, itching, shortness of breath, sweating, chills) were not present. In this patient, SU remained below BQL and the patient met responder criteria during Month 6 (exited study at Week 24). Anaphylaxis, cardiovascular event, and major adverse cardiovascular event (including non-fatal myocardial infarction, non-fatal stroke, cardiovascular death, and congestive heart failure) were not observed during any study period. Thirteen patients (92.9\%) experienced gout flares during treatment (2 severe flares). However, both the number of patients on treatment who flared and the frequency of flares decreased over time, from $4.2 \pm$ 2.3 flares (range: $1-8$ flares) in 13 of 14 patients (92.9\%) during the initial 12 weeks of treatment to $2.5 \pm$ 0.7 flares (range: $2-3$ flares) in 2 of 8 patients (25.0\%) during Weeks 37-52; Figure 2.

Liver and kidney function were carefully monitored during both the Run-in and Treatment periods. At Screening, 3 patients in the mITT population (21.4\%) had an ALT above the upper limit of normal (ULN) and 2 patients (14.3\%) had an AST above the ULN. A minor, transient increase in LFTs occurred shortly after methotrexate initiation, with LFTs stabilizing below the ULN at Week 2 (Figure 5a, b). LFTs remained stable through Week 52. Two patients experienced one mild (grade 1) LFT elevation, both of which resolved following a methotrexate dose reduction $(15 \mathrm{mg} /$ week to $10 \mathrm{mg} /$ week at Week 25 in 1 patient [titrated back to $15 \mathrm{mg} /$ week beginning at Week 37] and Week 2 in 1 patient). Methotrexate was otherwise well-tolerated in the mITT population. One patient had an accidental dose reduction to 12.5 $\mathrm{mg} /$ week on 2 occasions (Weeks 9 and 18). All 3 patients who had a methotrexate dose reduction remained treatment responders during the methotrexate/pegloticase treatment period.

Mean estimated glomerular filtration rate (eGFR) was $84.6 \pm 21.7 \mathrm{~mL} / \mathrm{min} / 1.73 \mathrm{~m}^{2}$ just prior to beginning methotrexate (Week $-4, n=14$ ) and remained stable throughout the methotrexate Run-in and methotrexate+pegloticase Treatment periods (Figure $5 \mathrm{c}$ ). Mean eGFR was $88.3 \pm 21.1 \mathrm{~mL} / \mathrm{min} / 1.73 \mathrm{~m}^{2}$ 
at Week 24 (change from Week $-4:+4.2 \pm 14.9 \mathrm{~mL} / \mathrm{min} / 1.73 \mathrm{~m}^{2}, \mathrm{n}=11$ ) and $80.9 \pm 22.4 \mathrm{~mL} / \mathrm{min} / 1.73 \mathrm{~m}{ }^{2}$ at Week 52 (change from Week $-4:-4.0 \pm 18.3 \mathrm{~mL} / \mathrm{min} / 1.73 \mathrm{~m}^{2}, \mathrm{n}=10$ ).

\section{Discussion}

Compared to previously reported responder rates for pegloticase monotherapy, an increased proportion of patients treated with methotrexate in conjunction with pegloticase maintained therapeutic response during Month 6 (42\% vs. 79\% [23]). Furthermore, all 6-month responders who remained on methotrexate+pegloticase therapy continued to be treatment responders through Month $12(\mathrm{SU}<1 \mathrm{mg} / \mathrm{dL}$ in all 8 patients). Duration of treatment response to pegloticase is of vital importance to patients with uncontrolled gout, many for whom pegloticase is their last medical treatment option.

The increased pegloticase treatment response rate is almost certainly attributable to the addition of methotrexate. Pegloticase is highly effective in lowering SU, but many patients have an incomplete response due to developing ADAs targeting polyethylene glycol moieties resulting in increased pegloticase clearance $[23,26]$. When compared to historical Phase 3 pivotal trial data, PK and ADA data from the current trial suggest that methotrexate reduces the immunogenicity of pegloticase and prolongs the biologic activity of pegloticase. Similar findings have been previously observed with other biologics (e.g., infliximab [39], adalimumab [40, 41], and certolizumab pegol [42]). Median pegloticase $C_{\text {min }}$ was higher $(1.03 \mu \mathrm{g} / \mathrm{mL}$ with methotrexate vs. BQL without methotrexate) and the proportion of patients with a pegloticase $C_{\min } B Q L(36 \%$ vs. $77 \%$ ) was lower in patients treated with methotrexate/pegloticase cotherapy than those treated with pegloticase monotherapy (the lowest quantitation limit was similar between studies). Further, 2 of 11 primary endpoint responders in the current trial developed low titre ( $\leq$ 40) anti-PEG antibodies at a single visit following pegloticase treatment (remaining 9 responders were ADA negative or did not exceed their baseline ADA levels). In contrast, 2 of the 3 non-responders developed ADA titres concomitantly with an undetectable serum pegloticase concentration and increase in SU above $6 \mathrm{mg} / \mathrm{dL}$. The remaining non-responder was positive for anti-PEG antibody titre prior to first pegloticase dose, but negative thereafter. Pre-infusion serum pegloticase concentration was undetectable throughout treatment, for reasons that remain unclear. It is worth noting that the detection of antibody formation is dependent on assay sensitivity and specificity and that the incidence of antibody positivity may be influenced by assay processing methodology. Therefore, comparison of antibody incidence in the current study to historical pegloticase studies may not be appropriate. Concentrations of MTX-PGs were maintained during the treatment course, suggesting compliance of methotrexate administration. Additionally, there was no apparent difference in MTX-PG concentrations between responders and nonresponders (data not shown).

Extended safety data indicate that methotrexate/pegloticase therapy was well tolerated over the 52-week treatment period and no new safety concerns over pegloticase monotherapy or the 6-month findings ${ }^{34}$ were identified. Gout flares remained the most common AE observed (13 of 14 patients [92.9\%]) and occurred in most patients during the first 12 weeks of pegloticase therapy. However, both the number of 
patients experiencing flares and the frequency of flares markedly decreased over time. On average, worsening of hepatic or renal function was not noted throughout the Run-in or Treatment periods. Two AEs of increased LFTs were observed. Both were graded as mild and resolved with methotrexate dose reduction. Other AEs that were observed in more than 1 patient during the Treatment period included diarrhoea, nasopharyngitis, upper respiratory tract infection, arthralgia, muscle strain, sinusitis, and hypertension.

This study was limited by its small size, open-label design, and lack of a control group. Larger, randomized, placebo-controlled trials are needed to more rigorously examine the benefits and associated risks of administering methotrexate in conjunction with pegloticase to patients with uncontrolled gout. Such a trial is underway (MIRROR RCT, NCT03994731). Finally, there remains the question if lower doses of methotrexate may be able to achieve similar successful results as those seen here.

\section{Conclusions}

In conclusion, these data further support the use of methotrexate with pegloticase in patients with uncontrolled gout. New pharmacokinetic and ADA data presented here strongly suggest that methotrexate reduces immunogenicity to pegloticase, allowing more patients to accomplish treatment goals from full courses of therapy with a reduction in previously seen adverse effects. This benefit seems to be sustained for at least 12 months in patients remaining on co-therapy with both pegloticase and methotrexate.

\section{Abbreviations}

SU: serum urate; ULT: urate-lowering therapy; IR: infusion reaction; ADA: anti-drug antibody; BQL: below quantification limits

\section{Declarations}

\section{Ethics approval and consent to participate}

This multicentre, open-label, efficacy and safety study (ClinicalTrials.gov: NCT03635957) was reviewed and approved for the 6 participating sites by the Western Institutional Review Board (Puyallup, WA; IRB assurance number: IRB00000533, protocol approval number 20182156). All patients provided written informed consent to participate and all study conduct adhered to the tenants of the Declaration of Helsinki.

\section{Consent for publication}

Not applicable.

\section{Availability of data and materials}


Horizon is committed to responsibly sharing data from the clinical trials we sponsor. Access to anonymized, individual, and trial-level data (analysis data sets) may be granted to qualified researchers for independent scientific research, provided the trials are not part of an ongoing or planned regulatory submission (including clinical trial data for unlicensed products and indications). Data may be requested by submitting a research proposal and Statistical Analysis Plan and will be provided following review and approval of the plan and execution of a Data Sharing Agreement. For more information, or to submit a request, please submit to: medicalinformation@horizontherapeutics.com.

\section{Competing interests}

JKB has received research support from Horizon Therapeutics and Radius Health as a study site and principal investigator. He has received consulting/speaker fees $>10 \mathrm{k}$ from Horizon Therapeutics, Celgene, Novartis, and AbbVie. JRPT has served as a consultant/advisory board member for BMS, Janssen, Lilly Pfizer, Sanofi-Genzyme, AbbVie, Aurinia, AstraZeneca, Samumed/Biosplice. He has served as a speaker for AbbVie, Amgen, BMS, Janssen, Lilly, Pfizer, Sanofi/Genzyme, Aurinia, AstraZeneca, GlaxoSmithKline. He has received research grants and support from AbbVie, Amgen, BMS, Boehringer Ingelheim, Genentech, Gilead, Horizon Therapeutics, Janssen, Lilly, Pfizer, Vorso, Samumed/Biosplice, Selecta, Exagen, CSL Behring, Organogenesis, SunPharma, DRL, and Emerald. RB has no conflicts of interest to disclose. HMK has received research support from Horizon Therapeutics (study site/investigator), is an advisor and speaker for Horizon Therapeutics, and is an owner and Chairmen of the Board of Discus Analytics (JoinMan). PMP, KO, YS, BL, LZ, YX, JC, and SR are employees of and own stock in Horizon Therapeutics. MEW has received grants from Amgen, Bristol-Myers Squibb, Lilly, and Sanofi. He has received consulting fees greater than $\$ 10,000$ USD from Chemocentryx, Corona, and Genosco and less than $\$ 10,000$ USD from AbbVie, Amgen, Aclaris, Arena, Bayer, Bristol Meyer Squibb, Crescendo Myriad Genetics, GlaxoSmithKline, Gilead Sciences, Horizon Therapeutics, Johnson and Johnson, Eli Lilly, Novartis, Pfizer, Rani Therapeutics, Roche, Samsung, Scipher Medicine, Set Point, Tremeau, and XBiotech; he has stock options in Can-Fite BioPharma, Scipher Medicine, Inmedix, and Vorso and royalties from Elsevier as co-editor for the textbook Rheumatology. JP has received research support from Horizon Therapeutics (study site/investigator). He has also served as an advisor and speaker for Horizon Therapeutics.

\section{Funding}

This work was supported by Horizon Therapeutics plc. Horizon was involved in study design and data collection, analysis, and interpretation. Horizon was also involved in manuscript drafting and editing.

\section{Authors' contributions}

JKB collected patient data, contributed to study design, and was a major contributor to manuscript writing. JRPT, RB, and HMK collected patient data and provided critical manuscript review. PMP contributed to study design, data interpretation, and was a major contributor to manuscript writing. KO, YS, YX, JC, and SR contributed to study design, analysed, and interpreted data, and were major 
contributors to manuscript writing. $\mathrm{BL}$ and $\mathrm{LZ}$ contributed to study design, data interpretation, and was a major contributor to manuscript writing. MEW contributed to study design and provided critical manuscript review. JP collected patient data, contributed to study design, and provided critical manuscript review. All authors have read and approved the final manuscript.

\section{Acknowledgements}

We acknowledge the following employees of Horizon Therapeutics: Lissa Padnick-Silver, Ph.D. and Megan Francis-Sedlak, Ph.D., for writing and editorial assistance, and Colleen Canavan, M.S. for trial support. We also acknowledge Exagen, Inc. for assistance with performing and interpreting MTX-PG measurements.

\section{References}

1. Park JJ, Roudier MP, Soman D, Mokadam NA, Simkin PA. Prevalence of birefringent crystals in cardiac and prostatic tissues, an observational study. BMJ Open 2014;4:e005308.

2. Kingsbury SR, Conaghan PG, McDermott MF. The role of the NLRP3 inflammasome in gout. J Inflamm Res 2011;4:39-49.

3. Choi HK, Ford ES, Li C, Curhan G. Prevalence of the metabolic syndrome in patients with gout: the Third National Health and Nurtrition Examination Survey. Arthritis Care Res (Hoboken) 2007, 57:10915.

4. Pan A, Teng GG, Yuan JM, Koh WP. Bidirectional association between self-reported hypertension and gout: The Singapore Chinese Health Study. PloS One 2015;10:e0141749.

5. Zhao G, Huang L, Song M, Song Y. Baseline serum uric acid level as a predictor of cardiovascular disease related mortality and all-cause mortality: a meta-analysis of prospective studies. Atherosclerosis 2013;231:61-8.

6. Nozue T, Yamamoto S, Tohyama S, Fukui K, Umezawa S, Onishi Y, et al. Correlations between serum uric acid and coronary atherosclerosis before and during statin therapy. Coron Art Dis 2014;25:343-8.

7. Tamariz L, Hernandez F, Bush A, Palacio A, Hare JM. Association between serum uric acid and atrial fibrillation: a systematic review and meta-analysis. Heart Rhythm 2014;11:1102-8.

8. Kim SY, Guevara JP, Kim KM, Choi HK, Heitjan DF, Albert DA. Hyperuricemia and risk of stroke: a systematic review and meta-analysis. Arthritis Rheum 2009;61:885-92.

9. Culleton BF, Larson MG, Kannel WB, Levy D. Serum uric acid and risk for cardiovascular disease and death: the Framingham Heart Study. Ann Intern Med 1999;131:7-13.

10. Pan A, Teng GG, Yuan JM, Koh WP. Bidirectional association between diabetes and gout: the Singapore Chinese Health Study. Sci Rep 2016;6:25766.

11. Roughley MJ, Belcher J, Mallen CD, Roddy E. Gout and risk of chronic kidney disease and nephrolithiasis: meta-analysis of observational studies. Arthritis Res Ther 2015;17:90. 
12. Yu KH, Kuo CF, Luo SF, See LC, Chou IJ, Chang HC, et al. Risk of end-stage renal disease associated with gout: a nationwide population study. Arthritis Res Ther 2012;14:R83.

13. Chen JH, Lan JL, Cheng CF, Liang WM, Lin HY, Tsay GJ, et al. Effect of Urate-Lowering Therapy on All-Cause and Cardiovascular Mortality in Hyperuricemic Patients without Gout. A Case-Matched Cohort Study. PloS One 2015;10:e0145193.

14. Choi HK, Curhan G. Independent impact of gout on mortality and risk for coronary heart disease. Circulation 2007;116:894-900.

15. FitzGerald JD, Dalbeth N, Mikuls T, Brignardello-Petersen R, Guyatt G, Abeles AM, et al. 2020 American College of Rheumatology Guideline for the Management of Gout. Arthritis Care Res (Hoboken) 2020;72:744-60.

16. Richette P, Doherty M, Pascual E, Barskova V, Becce F, Castaneda-Sanabria J, et al. 2016 updated EULAR evidence-based recommendations for the management of gout. Ann Rheum Dis 2017;76:2942.

17. Fels E, Sundy JS: Refractory gout: what is it and what to do about it? Current Opin Rheumatol 2008;20:198-202.

18. Riedel AA, Nelson M, Joseph-Ridge N, Wallace K, MacDonald P, Becker M. Compliance with allopurinol therapy among managed care enrollees with gout: a retrospective analysis of administrative claims. J Rheumatol 2004;31:1575-81.

19. Harrold LR, Andrade SE, Briesacher BA, Raebel MA, Fouayzi H, Yood RA, Ockene IS. Adherence with urate-lowering therapies for the treatment of gout." Arthritis Res Ther 11(2): R46.

20. Dalbeth N, Nicolaou S, Baumgartner S, Hu J, Fung M, Choi HK. Presence of monosodium urate crystal deposition by dual-energy CT in patients with gout treated with allopurinol. Ann Rheum Dis 2018;77:364-70.

21. Brook RA, Forsythe A, Smeeding JE, Lawrence Edwards N: Chronic gout. epidemiology, disease progression, treatment and disease burden. Curr Med Res Opin 2010;26:2813-21.

22. Becker MA, Schumacher HR, Benjamin KL, Gorevic P, Greenwald M, Fessel J, et al. Quality of life and disability in patients with treatment-failure gout. J Rheumatol 2009;36:1041-8.

23. Sundy JS, Baraf HS, Yood RA, Edwards NL, Gutierrez-Urena SR, Treadwell EL, et al. Efficacy and tolerability of pegloticase for the treatment of chronic gout in patients refractory to conventional treatment: two randomized controlled trials. JAMA 2011; 306:711-20.

24. Keenan RT, Baraf HSB, LaMoreaux B. Use of pre-infusion serum uric acid levels as a biomarker for infusion reaction risk in patients on pegloticase. Rheumatol Ther 2019, 6:299-304.

25. Baraf HS, Yood RA, Ottery FD, Sundy JS, Becker MA. Infusion-related reactions with pegloticase, a recombinant uricase for the treatment of chronic gout refractory to conventional therapy. J Clin Rheumatol 2014; 20:427-32.

26. Lipsky PE, Calabrese LH, Kavanaugh A, Sundy JS, Wright D, Wolfson M, et al. Pegloticase immunogenicity: the relationship between efficacy and antibody development in patients treated for refractory chronic gout. Arthritis Res Ther 2014;16:R60. 
27. Hershfield MS, Ganson NJ, Kelly SJ, Scarlett EL, Jaggers DA, Sundy JS. Induced and pre-existing anti-polyethylene glycol antibody in a trial of every 3-week dosing of pegloticase for refractory gout, including in organ transplant recipients. Arthritis Res Ther 2014;16:R63.

28. Botson JK, Peterson J. Pretreatment and coadministration with methotrexate improved durability of pegloticase response: an observational, proof-of-concept case series. J Clin Rheum [Epub ahead of print].

29. Albert JA, Hosey T, LaMoreaux B. Increased efficacy and tolerability of pegloticase in patients with uncontrolled gout co-treated with methotrexate: a retrospective study. Rheumatol Ther 2020;7:639648.

30. Masri K, Winterling K, LaMoreaux B. Leflunomide co-therapy with pegloticase in uncontrolled gout. Ann Rheum Dis 2020;79:450.

31. Rainey H, Baraf HSB, Yeo A, Lipsky P. Companion immunosuppression with azathioprine increases the frequency of persistent responsiveness to pegloticase in patients with chronic refractory gout. Ann Rheum Dis 2020;79:438.

32. Botson JK, Tesser JRP, Bennett R, Kenney HM, Obermeyer KO, LaMoreaux B, et al. Pegloticase in combination with methotrexate in patients with uncontrolled gout: a multicenter, open-label study (MIRROR). J Rheumatol 2021;48:767-774.

33. Khanna P, Khanna D, Cutter G, Foster J, Melnick J, Jaafar S, et al. Reducing immunogenicity of pegloticase with concomitant use of mycophenolate mofetil in patients with refractory gout: a phase II, randomized, double-blind, placebo-controlled trial. Arthritis Rheumatol 2021 [Epub ahead of print].

34. LaMoreaux B, Francis-Sedlak M, Svensson K, Holt R. Immunomodulation co-therapy with pegloticase: database trends 2014-2019 [abstract]. Ann Rheum Dis 2020;79(suppl 1):108.

35. Woodworth T, Furst DE, Alten R, Bingham CO 3rd, Yocum D, Sloan V, et al. Standardizing assessment and reporting of adverse effects in rheumatology clinical trials II: the Rheumatology Common Toxicity Criteria v.2.0. J Rheumatol 2007;34:1401-14.

36. Gaffo AL, Schumacher HR, Saag KG, Taylor WJ, Dinnella J, Outman R, et al. Developing a provisional definition of flare in patients with established gout. Arthritis Rheum 2012;64:1508-17.

37. Dervieux T, Orentas Lein D, Marcelletti J, Pischel K, Smith K, Walsh M, Richerson R. HPLC determination of erthrocyte methotrexate polyglutamates after low-dose methotrexate therapy in patients with rheumatoid arthritis. Clin Chem 2003;49:1632-1641.

38. Yue CS, Maroli AN, Lavigne JR, Huang W, Wright D, Waltrip R, et al. Population pharmacokinetic and pharmacodynamic analysis of pegloticase administered by intravenous infusion in two dose regimens to subjects with chronic gout. Clin Pharmacol Ther 2010;87:S90.

39. Maini RN, Breedveld FC, Kalden JR, Smolen JS, Davis D, Macfarlane JD, et al. Therapeutic efficacy of multiple intravenous infusions of anti-tumor necrosis factor alpha monoclonal antibody combined with low-dose weekly methotrexate in rheumatoid arthritis. Arthritis Rheum 1998;41:1552-63.

40. Humira (adalimumab) [package insert]. North Chicago, IL: AbbVie Inc.; 2021. 
41. Weisman MH, Moreland LW, Furst DE, Weinblatt ME, Keystone EC, Paulus HE, et al. Efficacy, pharmacokinetic, and safety assessment of adalimumab, a fully human anti-tumor necrosis factoralpha monoclonal antibody, in adults with rheumatoid arthritis receiving concomitant methotrexate: a pilot study. Clin Ther 2003;25:1700-21.

42. Cimzia (certolizumab pegol) [package insert]. Smyrna, GA: UCB, Inc.; 2016.

\section{Figures}

Figure 1

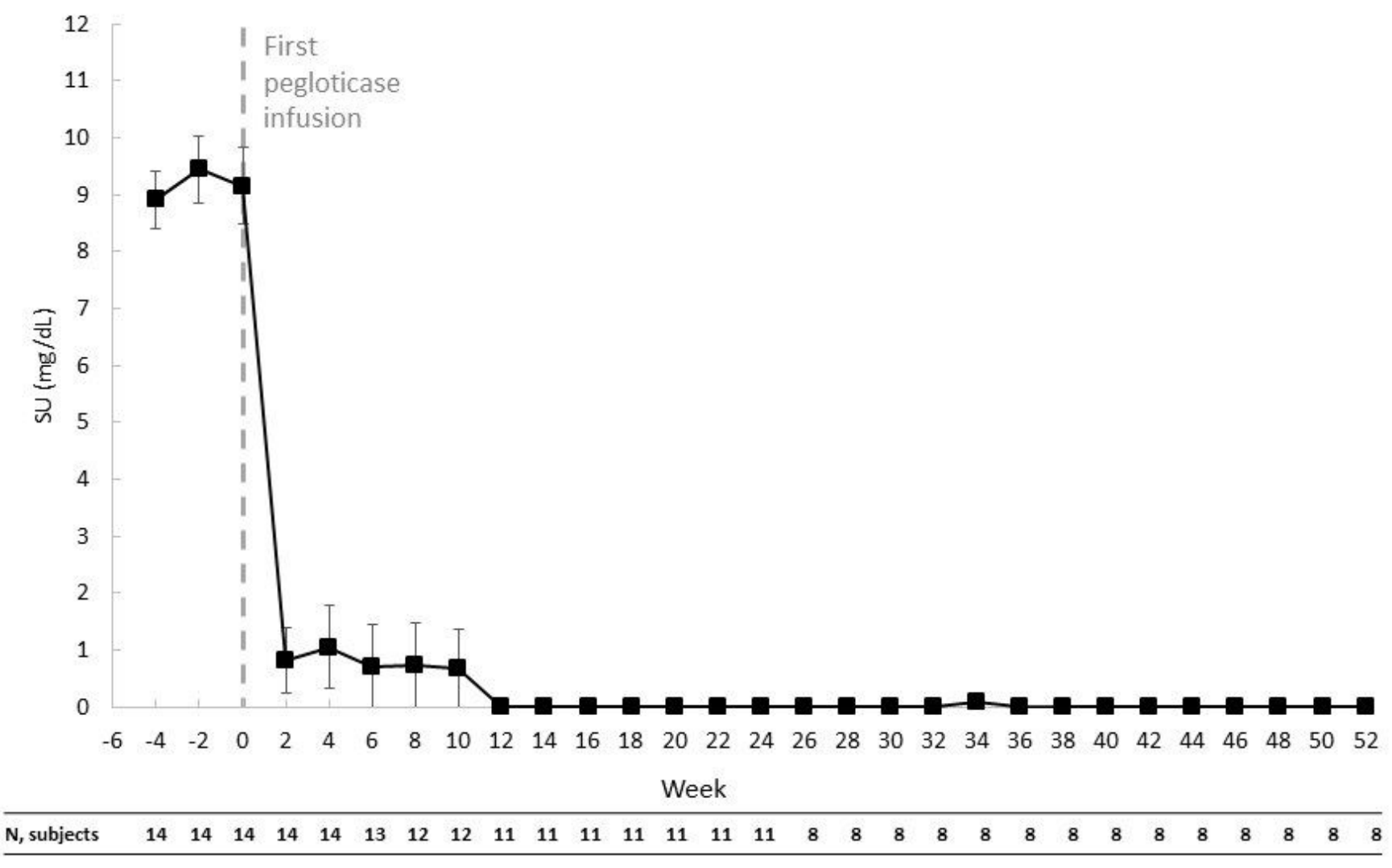

\section{Figure 1}

Pre-infusion serum urate levels during the methotrexate Run-in (4 weeks) and pegloticase+methotrexate treatment (up to 52 weeks) periods. In the 3 non-responders, serum urate (SU) increases above $6 \mathrm{mg} / \mathrm{dL}$ were noted at Weeks 2 and 4, Weeks 4 and 6, and Weeks 8 and 10. Data points represent mean values and error bars represent standard error (includes patients on treatment, values below the lower limit of detection were set to 0 ). SU, serum urate. 
Figure 2
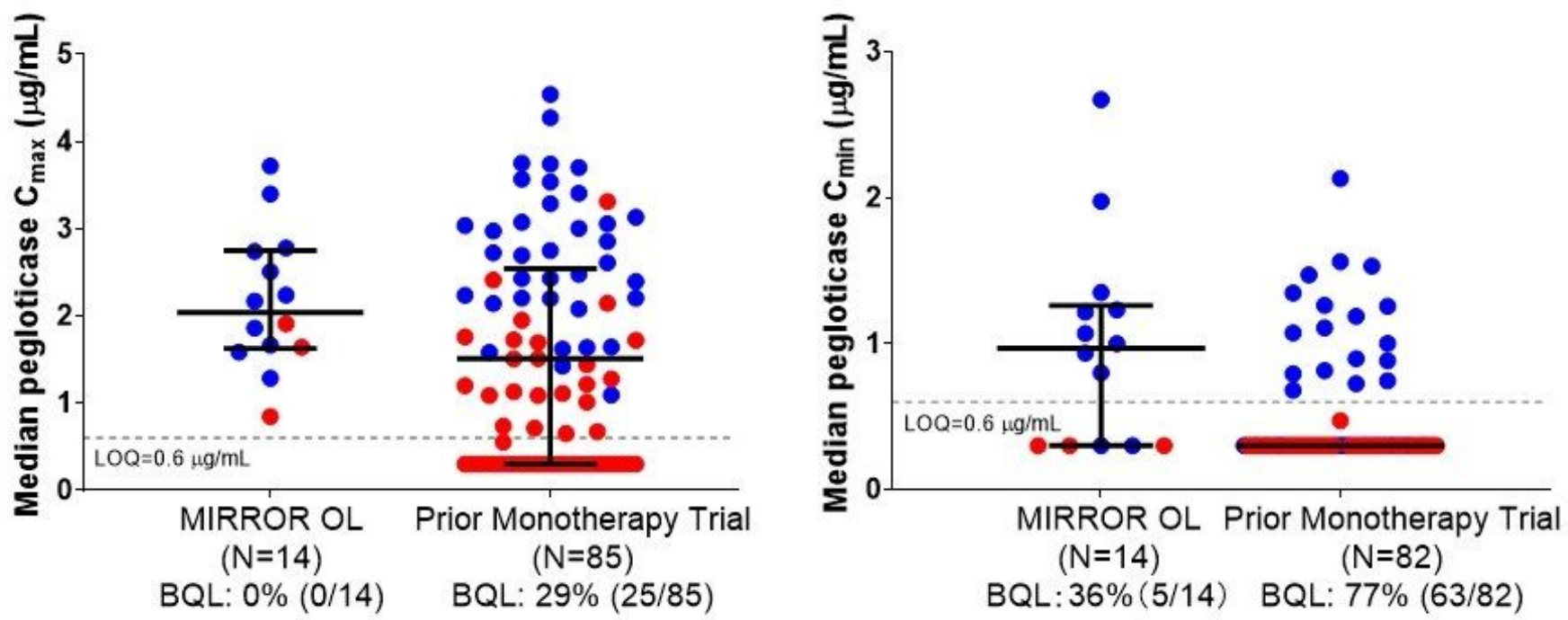

Figure 2

Comparison of pegloticase exposure with methotrexate co-treatment in current study (MIRROR OL) and as monotherapy in prior Phase 3 trials. Blue circles represent treatment responders and red circles represent non-responders. The gray dotted line shows the limit of quantitation limit (LOQ) of pegloticase measurements $(0.6 \mu \mathrm{g} / \mathrm{mL})$. Data below LOQ (BQL) were imputed as $0.3 \mu \mathrm{g} / \mathrm{mL}$. 
Figure 3

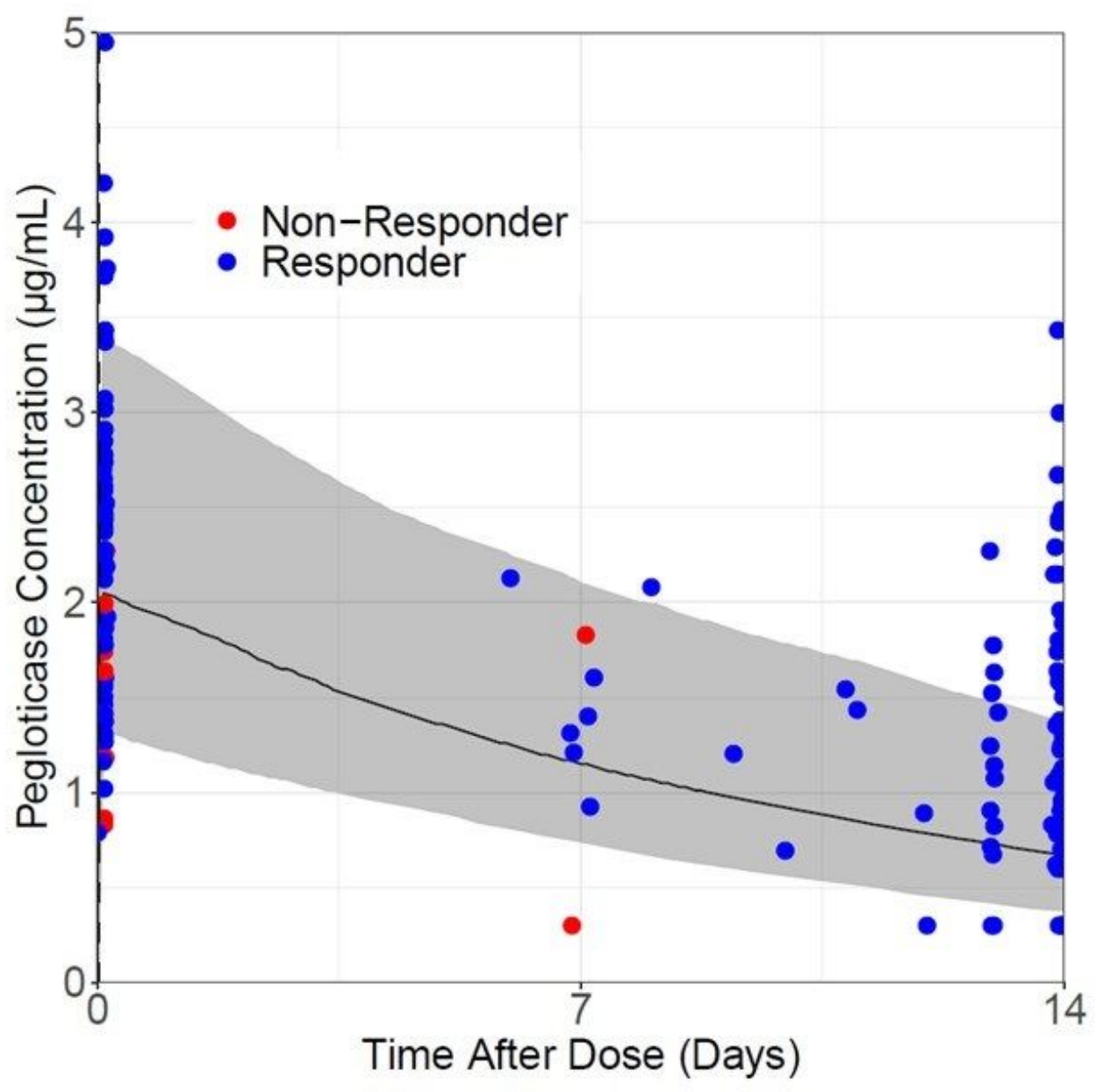

Figure 3

Comparison of observed pegloticase concentrations in the current study (MIRROR OL, pegloticase+methotrexate co-therapy) and in a simulated PK profile of prior Phase 3 trials (pegloticase monotherapy). Circles represent observed data in MIRROR OL with non-responders in red and responders in blue. Simulated monotherapy pegloticase concentration over time is shown as median concentration (black line) with $90 \%$ confidence intervals (gray shaded area). The simulation was modeled using time elapsed from the start of each infusion, pooling data from all 12 infusions administered to Phase 3 pivotal trial participants. 


\section{Figure 4}

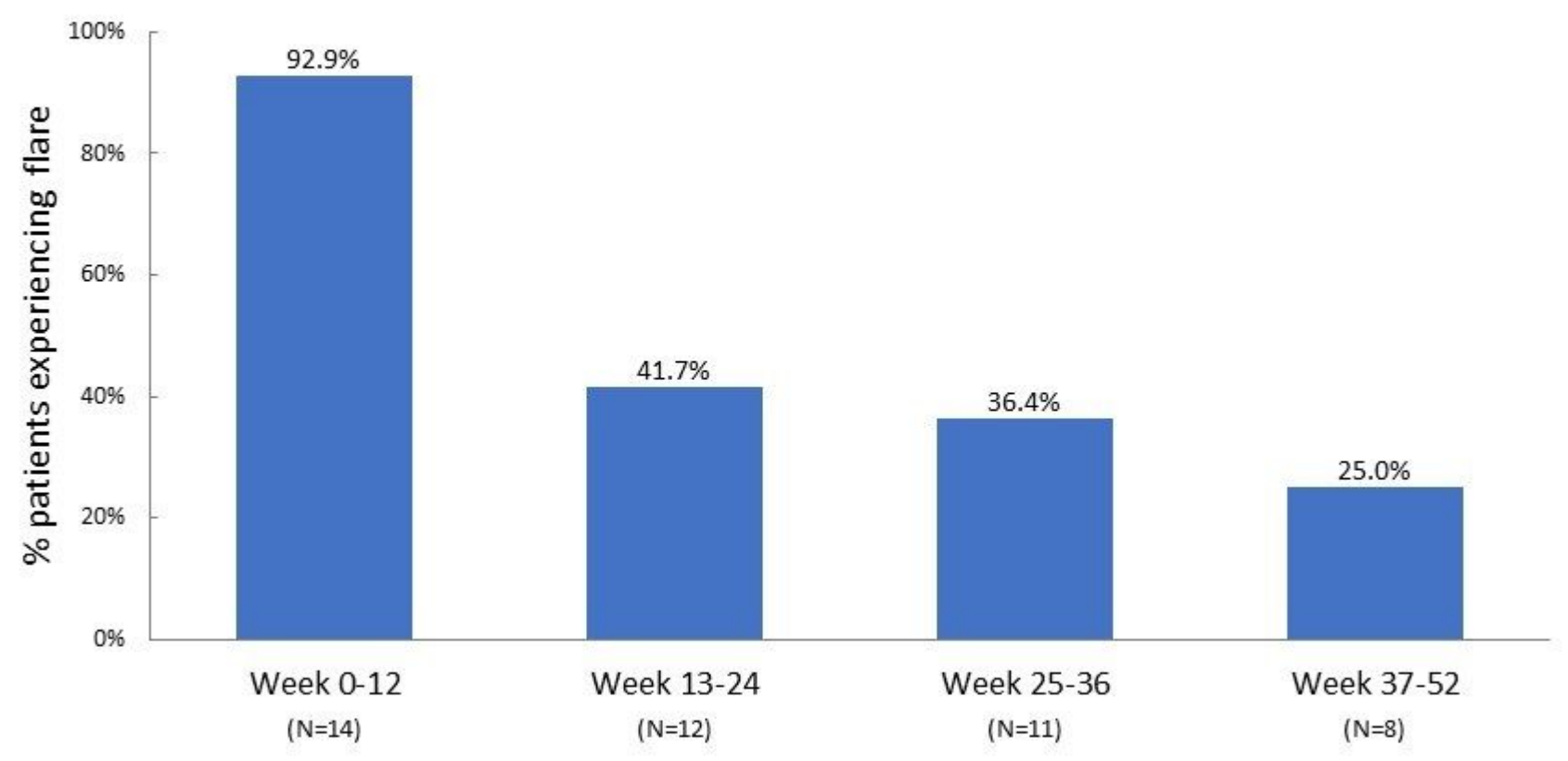

\section{Figure 4}

Proportion of patients on treatment experiencing gout flares during the methotrexate+pegloticase treatment period. Mean number of flares during Weeks $0-12$ and Weeks $37-52$ was $4.2 \pm 2.3$ (range: 1-8) and $2.5 \pm 0.7$ (range: $2-3$ ), respectively. 
Figure 5
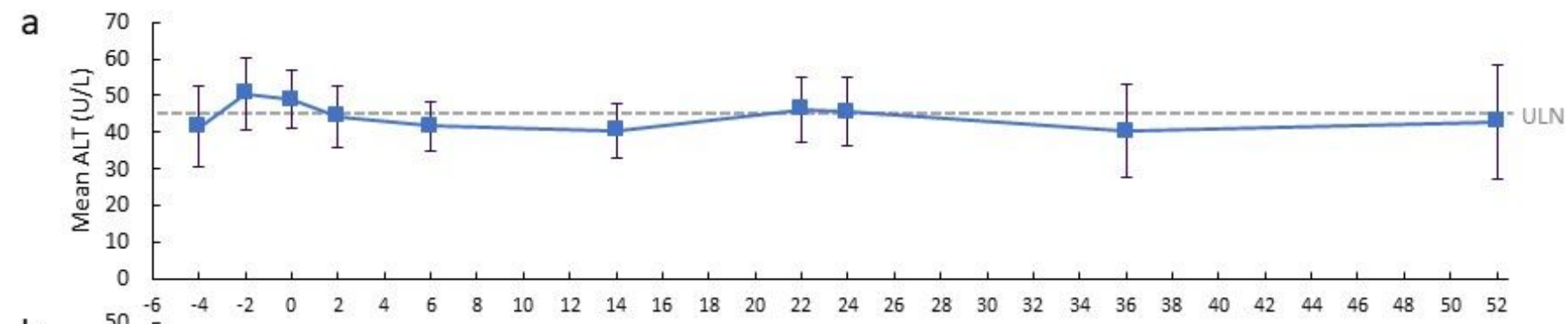

b

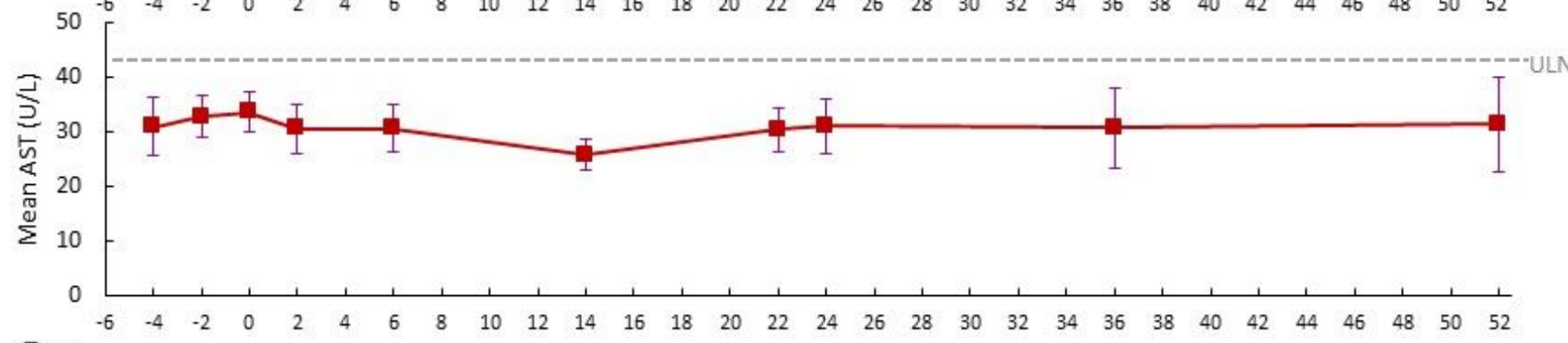

C

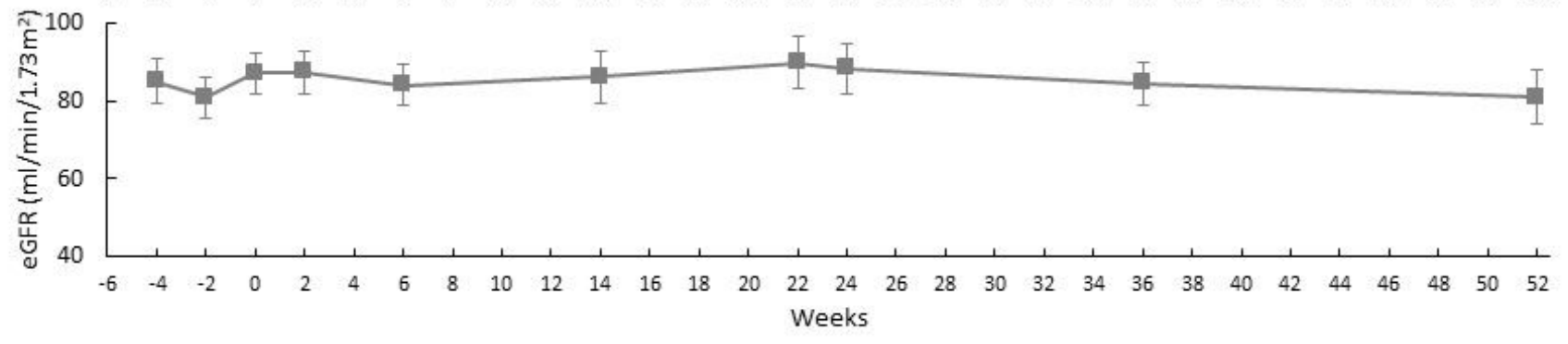

\begin{tabular}{lllllllllll}
\hline d & Number of subjects & 14 & 14 & 14 & 14 & 14 & 11 & 11 & 11 & 10 \\
\hline Number with ALT $>$ ULN & 3 & 5 & 6 & 4 & 5 & 5 & 5 & 5 & 2 & 2 \\
Number with AST > ULN & 2 & 4 & 2 & 1 & 3 & 1 & 3 & 2 & 2 & 2 \\
Number with EGFR $\leq 60$ & 2 & 2 & 1 & 2 & 2 & 1 & 1 & 2 & 1 \\
\hline
\end{tabular}

\section{Figure 5}

Liver ( $(a, b)$ and renal (c) function test results through the Run-in (Week -4 to 0) and Treatment (Week 0 to 52) periods. Week -4 values were measured prior to methotrexate exposure. Week 0 (Day 1 ) values were measured prior to pegloticase exposure. The number of patients with liver function tests above the upper limits of normal and estimated glomerular filtration rate $<60 \mathrm{~mL} / \mathrm{min} / 1.73 \mathrm{~m} 2$ are also shown (d). Error bars represent standard error. ALT, alanine aminotransferase; AST, aspartate aminotransferase; eGFR, estimated glomerular filtration rate (calculated from serum creatinine measurements using the MDRD equation).

\section{Supplementary Files}

This is a list of supplementary files associated with this preprint. Click to download.

- Additionalfile1.docx

- Additionalfile2.pptx 
- Additionalfile3.pptx

Page 24/24 